\title{
Approximation by univariate, bivariate and GBS operators of Chlodowsky-Kantorovich operators
}

Harun Cicek, Resat Aslan and Aydin Izgi

Department of Mathematics, Harran University, Şanlıurfa, Turkey

Received: 19 February 2020, Accepted: 22 June 2020

Published online: 25 June 2020.

\begin{abstract}
We define univariate, bivariate and GBS operators of Chlodowsky-Kantorovich operators and we give their approximations and rate of their approximations for B-continuous and B-differentiable functions. Finally we give some plots to compare operators and their GBS operators.
\end{abstract}

Keywords: GBS-operators of Chlodowsky-Kantorovich operators, Bögel continuous, Bögel differentiable, rate of approximation, bivariate functions.

\section{Introduction and the construct of the one variable Chlodowsky-Kantorovich operators}

Classical approximation theory, including polynomial approximation is a fundamental research area in applied mathematics. Development in approximation theory plays an important role in numerical solution of partial differential equations, image processing as well as in data sciences and many other disciplines. For example, radial basis functions and shift-invariant spaces are widely used for geometric modeling in aerospace and automobile industries. Due to the importance of polynomials, a variety of their generalizations and related topics have been studied in [1],[2], [3] and [4].

Bernstein polynomials are not useful for discontinuous measurable functions. More suitable modification of the Bernstein polynomials, for this kind functions, introduced by Kantorovich [5] : For any $f \in L_{p}[0,1], x \in[0,1]$ and $p \geq 1$

$$
K_{n}(f ; x)=(n+1) \sum_{k=0}^{n} \varphi_{n}^{k}(x) \int_{\frac{k}{n+1}}^{\frac{k+1}{n+1}} f(t) d t .
$$

(1) If we take definite integral operator of $f$ as

$$
F(x)=\int_{0}^{x} f(t) d t
$$

then it is possible to connect the Kantorovich polynomials with the Bernstein polynomials by means of the identity

$$
K_{n}(f ; x)=\frac{d}{d x} B_{n+1}(f ; x)
$$


(see [3], cf:30). Chlodowsky [6] modified Bernstein operators as the following:

$$
C_{n}(f ; x)=\sum_{k=0}^{n} f\left(\frac{k}{n} b_{n}\right) \varphi_{n}^{k}\left(\frac{x}{b_{n}}\right), 0 \leq x \leq b_{n}
$$

where $\left(b_{n}\right)$ is a sequence with positive terms which increasing and satisfying the conditions

$$
\lim _{n \rightarrow 0} b_{n}=\infty \quad, \lim _{n \rightarrow 0} \frac{b_{n}}{n}=0
$$

Bernstein-Chlodowsky polynomials are studied in (for instance [7], [8], [9], [10]).

Chlodowsky-Kantorovich operators were introduced by Habib and Wafi in [11]. We produced our study by making use of the "Degree of Approximation of Functions by Modified Bernstein Polynomials On Unbounded Interval" article studied by Habib and Wafi in [11].

In this article we intend to study the approximation features of the operator

$$
H_{n}(f ; x)=\frac{(n+1)}{b_{n}} \sum_{k=0}^{n} \varphi_{n}^{k}\left(\frac{x}{b_{n}}\right) \int_{\frac{k b_{n}}{n+1}}^{\frac{(k+1) b_{n}}{n+1}} f(t) d t .
$$

which is Chlodowsky-Kantorovich operators. Here $\varphi_{n}^{k}(x)=\left(\begin{array}{c}n \\ k\end{array}\right) x^{k}(1-x)^{n-k}, x \in\left[0, b_{n}\right], f \in C\left[0, b_{n}\right]$ and $b_{n}$ is an increasing and positive sequence with features $\lim _{n \rightarrow 0} b_{n}=\infty, \lim _{n \rightarrow 0} \frac{b_{n}^{2}}{n}=0$ instead of (3).

Lemma 1. Let $e_{i}:=e_{i}(x)=x^{i}, E_{i}:=E_{i}(u, x)=(u-x)^{i}$ and $f \in C\left[0, b_{n}\right]$, we have

$H_{n}\left(e_{0} ; x\right)=1$,

$H_{n}\left(e_{1} ; x\right)=x+\frac{b_{n}-2 x}{2(n+1)}$,

$H_{n}\left(e_{2} ; x\right)=x^{2}+\frac{x\left[2 n b_{n}-(3 n+1) x\right]}{(n+1)^{2}}+\frac{b_{n}^{2}}{3(n+1)^{2}}$,

$H_{n}\left(e_{3} ; x\right)=x^{3}-\frac{6 n^{2}+n+1}{(n+1)^{3}} x^{3}+\frac{9 n(n-1) b_{n}}{2(n+1)^{3}} x^{2}+\frac{15 n b_{n}^{2}}{2(n+1)^{3}} x+\frac{b_{n}^{3}}{4(n+1)^{3}}$

$=x^{3}+\frac{x}{2(n+1)^{3}}\left[15 n b_{n}^{2}+9 n(n-1) b_{n} x-\left(12 n^{2}+2 n+2\right) x^{2}\right]+\frac{b_{n}^{3}}{4(n+1)^{3}}$,

$H_{n}\left(e_{4} ; x\right)=x^{4}-\frac{10 n^{3}-5 n^{2}+10 n+1}{(n+1)^{4}} x^{4}+\frac{8 n(n-1)(n-2) b_{n}}{(n+1)^{4}} x^{3}+\frac{15 n(n-1) b_{n}^{2}}{(n+1)^{4}} x^{2}+\frac{38 n b_{n}^{3}}{(n+1)^{4}} x+\frac{b_{n}^{4}}{5(n+1)^{4}}$

$=x^{4}+\frac{x}{(n+1)^{4}}\left[38 n b_{n}^{3}+15 n(n-1) b_{n}^{2} x+8 n(n-1)(n-2) b_{n} x^{2}-\left(10 n^{3}-5 n^{2}+10 n+1\right) x^{3}\right]+\frac{b_{n}^{4}}{5(n+1)^{4}}$

From Lemma 1 we get the following equalities and inequalities:

$$
H_{n}\left(E_{2} ; x\right)=\frac{(n-1)}{(n+1)^{2}} x\left(b_{n}-x\right)+\frac{b_{n}^{2}}{3(n+1)^{2}},
$$




$$
H_{n}\left(E_{4} ; x\right)=\frac{3 n^{2}-20 n+1}{(n+1)^{4}} x^{4}-\frac{6 n^{2}-40 n+2}{(n+1)^{4}} b_{n} x^{3}+\frac{13 n^{2}+41 n+2}{(n+1)^{4}} b_{n}^{2} x^{2}+\frac{37 n-1}{(n+1)^{4}} b_{n}^{3} x+\frac{b_{n}^{4}}{5(n+1)^{4}}
$$

It is easy to see that on $\left[0, b_{n}\right]$

$$
H_{n}\left(E_{2} ; x\right) \leq \frac{3 n+1}{12(n+1)^{2}} b_{n}^{2}<\frac{b_{n}^{2}}{4(n+1)}
$$

and

$$
\begin{aligned}
H_{n}\left(E_{4} ; x\right) & \leq \frac{\left(3 n^{2}-20 n+1\right)+\left(13 n^{2}+41 n+2\right)+(37 n-1)+\frac{1}{5}}{(n+1)^{4}} b_{n}^{4} \\
& <\frac{16 n^{2}+58 n+3}{(n+1)^{4}} b_{n}^{4} \\
& <\frac{29}{(n+1)^{2}} b_{n}^{4} .
\end{aligned}
$$

And also it is seen that on $[0, A] \subset\left[0, b_{n}\right]$

$$
\begin{aligned}
H_{n}\left(E_{2} ; x\right) & <\frac{(n-1)}{(n+1)^{2}} A+\frac{b_{n}^{2}}{3(n+1)^{2}}=\frac{3(n-1) b_{n} A+b_{n}^{2}}{(n+1)^{2}} \\
& <\frac{\left(3 n A+b_{n}\right) b_{n}}{3(n+1)^{2}}<\frac{b_{n}}{(n+1)},
\end{aligned}
$$

and

$$
H_{n}\left(E_{4} ; x\right)<\frac{29}{(n+1)^{2}} b_{n}^{4}
$$

Theorem 1. Let $x \in\left[0, b_{n}\right], f \in C\left[0, b_{n}\right]$ and $H_{n}(f ; x)$ be linear positive operators.If

(i) $\lim _{n \longrightarrow \infty} H_{n}\left(e_{0} ; x\right)=1$

(ii) $\lim _{n \rightarrow \infty} H_{n}\left(e_{1} ; x\right)=x$

(iii) $\lim _{n \rightarrow \infty} H_{n}\left(e_{2} ; x\right)=x^{2}$

$H_{n}$ converges to $f$, for $f \in C\left[0, b_{n}\right]$.

From Lemma 1, (3) and defined feature $b_{n}$, the result is easily visible. Due to this theorem, $H_{n}(f ; x)$ operators fulfills the Korovkin condition.

\section{The construct of the bivariate operators of Chlodowsky-Kantorovich type}

In this section first we construct the bivariate operators of Chlodowsky-Kantorovich type on rectangle. Bivariate operators of Kantorovich type on simplex was introduced in ([26]).

The purpose of this paper is to give a representation for the bivariate Chlodowsky-Kantorovich operators.

For $n, m \in \mathbb{N}$ we define the operator $H_{n, m}: C\left(\left[0, b_{n}\right] \times\left[0, b_{m}\right]\right) \rightarrow C\left(\left[0, b_{n}\right] \times\left[0, b_{m}\right]\right)$ be defined for any function $f \in C\left(\left[0, b_{n}\right] \times\left[0, b_{m}\right]\right)$ by

$$
H_{n, m}(f ; x, y)=\frac{(n+1)(m+1)}{b_{n} b_{m}} \sum_{k=0}^{n} \sum_{j=0}^{m} \varphi_{n}^{k}\left(\frac{x}{b_{n}}\right) \varphi_{m}^{j}\left(\frac{y}{b_{m}}\right) \int_{\frac{k b_{n}}{n+1}}^{\frac{(k+1) b_{n}}{n+1}} \int_{\frac{j b_{m}}{m+1}}^{\frac{(j+1) b_{m}}{m+1}} f(t, u) d t d u
$$


for any $(x, y) \in\left[0, b_{n}\right] \times\left[0, b_{m}\right]$.

The operators defined above are called the bivariate operators of Chlodowsky-Kantorovich type. Clearly, the bivariate operators of Chlodowsky-Kantorovich type are linear and positive. And also we can see that easily

$$
H_{n, m}\left(t^{r} u^{s} ; x, y\right)=H_{n}\left(t^{r}, x\right) H_{m}\left(u^{s}, y\right) \text { and } H_{n, m}\left(t^{r}+u^{s} ; x, y\right)=H_{n}\left(t^{r}, x\right)+H_{m}\left(u^{s}, y\right)
$$

From Lemma 1 we can give the following lemma:

Lemma 2. Let $e_{i j}:=e_{i j}(x, y)=x^{i} y^{j}, E_{i j}:=E_{i j}(u, v ; x, y)=(u-x)^{i}(v-y)^{j}$ and $f \in C\left(\left[0, b_{n}\right] \times\left[0, b_{m}\right]\right)$, we have

$$
\begin{aligned}
& H_{n, m}\left(e_{00} ; x, y\right)=1 \\
& H_{n, m}\left(e_{10} ; x, y\right)=x+\frac{b_{n}-2 x}{2(n+1)}, H_{n, m}\left(e_{01} ; x, y\right)=y+\frac{b_{m}-2 y}{2(m+1)} \\
& H_{n, m}\left(e_{20} ; x, y\right)=x^{2}+\frac{x\left[2 n b_{n}-(3 n+1) x\right]}{(n+1)^{2}}+\frac{b_{n}^{2}}{3(n+1)^{2}} \\
& H_{n, m}\left(e_{02} ; x, y\right)=y^{2}+\frac{y\left[2 m b_{m}-(3 m+1) y\right]}{(m+1)^{2}}+\frac{b_{m}^{2}}{3(m+1)^{2}} \\
& H_{n, m}\left(e_{30} ; x, y\right)=x^{3}-\frac{6 n^{2}+n+1}{(n+1)^{3}} x^{3}+\frac{9 n(n-1) b_{n}}{2(n+1)^{3}} x^{2}+\frac{15 n b_{n}^{2}}{2(n+1)^{3}} x+\frac{b_{n}^{3}}{4(n+1)^{3}} \\
& H_{n, m}\left(e_{03} ; x, y\right)=y^{3}-\frac{6 m^{2}+m+1}{(m+1)^{3}} y^{3}+\frac{9 m(m-1) b_{m}}{2(m+1)^{3}} y^{2}+\frac{15 m b_{m}^{2}}{2(m+1)^{3}} y+\frac{b_{m}^{3}}{4(m+1)^{3}} \\
& H_{n, m}\left(e_{40} ; x, y\right)=x^{4}-\frac{10 n^{3}-5 n^{2}+10 n+1}{(n+1)^{4}} x^{4}+\frac{8 n(n-1)(n-2) b_{n}}{(n+1)^{4}} x^{3}+\frac{15 n(n-1) b_{n}^{2}}{(n+1)^{4}} x^{2}+\frac{38 n b_{n}^{3}}{(n+1)^{4}} x+\frac{b_{n}^{4}}{5(n+1)^{4}} \\
& =x^{4}+\frac{x}{(n+1)^{4}}\left[38 n b_{n}^{3}+15 n(n-1) b_{n}^{2} x+8 n(n-1)(n-2) b_{n} x^{2}\right. \\
& \left.-\left(10 n^{3}-5 n^{2}+10 n+1\right) x^{3}\right]+\frac{b_{n}^{4}}{5(n+1)^{4}} \\
& H_{n, m}\left(e_{04} ; x, y\right)=y^{4}-\frac{10 m^{3}-5 m^{2}+10 m+1}{(m+1)^{4}} y^{4}+\frac{8 m(m-1)(m-2) b_{m}}{(m+1)^{4}} y^{3}+\frac{15 m(m-1) b_{m}^{2}}{(m+1)^{4}} y^{2}+\frac{38 m b_{m}^{3}}{(m+1)^{4}} y+\frac{b_{m}^{4}}{5(m+1)^{4}} \\
& =y^{4}+\frac{y}{(m+1)^{4}}\left[38 m b_{m}^{3}+15 m(m-1) b_{m}^{2} y+8 m(m-1)(m-2) b_{m} y^{2}\right. \\
& \left.-\left(10 m^{3}-5 m^{2}+10 m+1\right) y^{3}\right]+\frac{b_{m}^{4}}{5(m+1)^{4}} .
\end{aligned}
$$

From Lemma 2, we have

$$
\begin{aligned}
& H_{n, m}\left(E_{20} ; x, y\right)=\frac{(n-1)}{(n+1)^{2}} x\left(b_{n}-x\right)+\frac{b_{n}^{2}}{3(n+1)^{2}}, \\
& H_{n, m}\left(E_{02} ; x, y\right)=\frac{(m-1)}{(m+1)^{2}} y\left(b_{m}-y\right)+\frac{b_{m}^{2}}{3(m+1)^{2}}
\end{aligned}
$$


and

$$
\begin{gathered}
H_{n, m}\left(E_{40} ; x, y\right)=\frac{3 n^{2}-20 n+1}{(n+1)^{4}} x^{4}-\frac{6 n^{2}-40 n+2}{(n+1)^{4}} b_{n} x^{3}+\frac{13 n^{2}+41 n+2}{(n+1)^{4}} b_{n}^{2} x^{2}+\frac{37 n-1}{(n+1)^{4}} b_{n}^{3} x+\frac{b_{n}^{4}}{5(n+1)^{4}}, \\
H_{n, m}\left(E_{04} ; x, y\right)=\frac{3 m^{2}-20 m+1}{(m+1)^{4}} y^{4}-\frac{6 m^{2}-40 m+2}{(m+1)^{4}} b_{m} y^{3}+\frac{13 m^{2}+41 m+2}{(m+1)^{4}} b_{m}^{2} y^{2}+\frac{37 m-1}{(m+1)^{4}} b_{m}^{3} y+\frac{b_{m}^{4}}{5(m+1)^{4}} .
\end{gathered}
$$

Theorem 2. Let $(x, y) \in\left[0, b_{n}\right] \times\left[0, b_{m}\right]$. and $f \in C\left(\left[0, b_{n}\right] \times\left[0, b_{m}\right]\right) ; H_{n, m}(f ; x, y)$ be linear positive operators. If

(i) $\lim _{n, m \longrightarrow \infty} H_{n}\left(e_{00} ; x, y\right)=1$

(ii) $\left.\lim _{n \rightarrow \infty} H_{n}\left(e_{10} ; x, y\right)\right)=x$

(iii) $\lim _{n, m \rightarrow \infty} H_{n}\left(e_{01} ; x, y\right)=y$

(iv) $\lim _{n, m \longrightarrow \infty} H_{n}\left(\left(e_{20}+e_{02} ; u, v\right)=x^{2}+y^{2} H_{n, m}\right.$ converges to $f$, for $f \in C\left(\left[0, b_{n}\right] \times\left[0, b_{m}\right]\right)$.

From Lemma 2, (3) and defined feature $b_{n}$, the result is easily visible. Due to this theorem, $H_{n, m}(f ; x, y)$ operators fulfills the Korovkin condition.

\section{Main results}

GBS operators of Chlodowsky-Kantorovich type, to establish a convergence theorem for these operators. We also give an approximation theorem for these operators in terms of the first modulus of smoothness and of the mixed modulus of smoothness for bivariate functions.

The terms of "B-Continuity" and "B-Differentiability" (Bögel-Continuity and Bögel-Differentiability) were introduced by K.Bögel ([13], [14], [15]) as the following. Let $X$ and $Y$ be compact real intervals, $f: X \times Y \rightarrow \mathbb{R}$ a function ,and $\Delta f\left[(x, y),\left(x_{0}, y_{0}\right)\right]=f(x, y)-f\left(x_{0}, y\right)-f\left(x, y_{0}\right)+f\left(x_{0}, y_{0}\right) . \Delta f\left[(x, y),\left(x_{0}, y_{0}\right)\right]$ is called mixed difference of $f$.

Definition 1. (a) A function $f$ is called a B-Continuous function in $\left(x_{0}, y_{0}\right) \in X \times Y$ if

$$
\lim _{(x, y) \rightarrow\left(x_{0}, y_{0}\right)} \Delta f\left[(x, y),\left(x_{0}, y_{0}\right)\right]=0 .
$$

(b) A function $f$ is called a B-Differentiable function in $\left(x_{0}, y_{0}\right) \in X \times Y$ if the following limit is exist and finite,

$$
\lim _{(x, y) \rightarrow\left(x_{0}, y_{0}\right)} \frac{\Delta f\left[(x, y),\left(x_{0}, y_{0}\right)\right]}{(x-y)\left(x_{0}-y_{0}\right)} .
$$

This B-Differentiable of $f$ in $\left(x_{0}, y_{0}\right)$ is denoted by $D_{B} f\left(x_{0}, y_{0}\right)$.

Let $F$ be the class of all functions $f: X \times Y \rightarrow \mathbb{R}$. Then we use subsets of $F$ which are given in the following:

$$
B(X \times Y)=\{f \in F: f \text { bounded on } X \times Y\}
$$

with usual sup-norm \|\|$_{\infty}$.

$$
B_{b}(X \times Y)=\left\{f \in F:\left|\Delta f\left[(x, y),\left(x_{0}, y_{0}\right)\right]\right| \leq K, \text { on } X \times Y, K>0\right\}
$$


is called $B$-bounded functions class with the norm

$$
\begin{gathered}
\|\|_{b}=\sup _{(x, y),\left(x_{0}, y_{0}\right) \in X \times Y}\left|\Delta f\left[(x, y),\left(x_{0}, y_{0}\right)\right]\right| . \\
C_{b}(X \times Y)=\{f \in F: f \text { is } B-\text { Continuous on } X \times Y\}, \\
D_{b}(X \times Y)=\{f \in F: f \text { is } B-\text { Differentable on } X \times Y\}
\end{gathered}
$$

The term GBS(Generalized Boolean Sum) operators were introduced by Badea and Kottin as the following [16].

An important theorem (the analogous of the well-known Korovkin theorem), for approximation of $B$-Continuous functions using GBS-operators is due to C. Badea and his collaborates [17].

In order to estimate the order approximation of $B$-Continuous functions using GBS operators, we use "the mixed modulus of smoothness" which was introduced by I. Badea [18]. It is analogous of first modulus of smoothness for univariate functions.

Definition 2. Let $f \in B_{b}(X \times Y)$. For any $\left(\delta_{1}, \delta_{2}\right) \in \mathbb{R}_{0,+}^{2}$, the mixed modulus of smoothness is the function $\omega_{\text {mixed }}\left(f ; \delta_{1}, \delta_{2}\right): \mathbb{R}_{0,+}^{2} \rightarrow \mathbb{R}$ defined by

$$
\omega_{\text {mixed }}\left(f ; \delta_{1}, \delta_{2}\right)=\sup \left\{|\Delta f[(x, y),(u, v)]|:|u-x| \leq \delta_{1},|v-y| \leq \delta_{2}\right\}
$$

where $\mathbb{R}_{0,+}:=[0, \infty)$.

The features of mixed moduli of continuity ;

$$
\omega_{\text {mixed }}\left(f ; \lambda_{1} \delta_{1}, \lambda_{1} \delta_{2}\right) \leq\left(1+\lambda_{1}\right)\left(1+\lambda_{2}\right) \omega_{\text {mixed }}\left(h ; \delta_{1}, \delta_{2}\right)
$$

we can write,

$$
\begin{aligned}
|\nabla h[(u, v) ;(x, y)]| & \leq \omega_{\text {mixed }}(f ;|u-x|,|v-y|) \\
& \leq\left(1+\frac{|u-x|}{\delta_{1}}\right)\left(1+\frac{|v-y|}{\delta_{2}}\right) \omega_{\text {mixed }}\left(f ; \delta_{1}, \delta_{2}\right)
\end{aligned}
$$

There are many studies about approximation by associated GBS operators of some well-known operators and their generalizations. For instance, ([18]-[19]), ([20]-[22]) and [23].

The approximation theorems for bivariate functions were first given by Volkov in [24] and approximation of the GBS operators of associate with operators of two variables were established by [17]. More detail about GBS it can be seen in ([16], [25], [13], [14], [15], [21] ).

Now from (11) we construct GBS operator associated to the operator $H_{n, m}$ :

$$
H_{n, m}^{*}(f ; x, y)=H_{n, m}((f(t, y)+f(x, u)-f(t, u)) ; x, y)
$$

Define $D_{A B}=[0, A] \times[0, B]$ where $[0, A] \times[0, B] \subset\left[0, b_{n}\right] \times\left[0, b_{m}\right]$. 
In the following theorems we examine approximation of $\left(P_{n, m}^{*}\right)_{n, m \in \mathbb{N}}$ to $f$ (here $f \in C_{b}\left(D_{A B}\right)$ or $\left.f \in D_{b}\left(D_{A B}\right)\right)$ and rate of approximation of $\left(P_{n, m}^{*}\right)_{n, m \in \mathbb{N}}$ to $f$. Taking into account the relations theorems defined by Bögel we obtain the following theorem.

Theorem 3. The sequence $\left(H_{n, m}^{*}\right)_{n, m \in \mathbb{N}}$ converges to any $f \in C\left(D_{A B}\right)$ uniformly. It easy to see the following relation:

$$
H_{n, m}^{*}\left(t^{i} u^{j} ; x, y\right)=x^{i} y^{j} \text { for all } i, j=0,1,2, \ldots
$$

That means there is no approximation for any usual continuous function $f$ on $[0, A]$. Mean $H_{n, m}^{*}(f ; x, y)=f$ for all $f \in C\left(D_{A B}\right)$.

Theorem 4. If $f \in C_{b}\left(D_{b_{n} b_{m}}\right)$, then for any $(x, y) \in R_{A B}$, the following relation holds for all :

$$
\left|H_{n, m}^{*}(f(u, v) ; x, y)-f(x, y)\right| \leq 3 . \omega_{\text {mixed }}\left(f ; \sqrt{\frac{b_{n}}{(n+1)}}, \sqrt{\frac{b_{m}}{(m+1)}}\right) .
$$

Proof. Applying Theorem given in [16] for rate of GBS operators, Lemma 2 ,( by taking in account (4),(11), (19) and (20) we have

$$
\begin{aligned}
\left|H_{n, m}^{*}(f(u, v) ; x, y)-f(x, y)\right| \leq & \left(\frac{1}{\delta_{1}} \sqrt{H_{n, m}\left(E_{20} ; x, y\right)}+\frac{1}{\delta_{2}} \sqrt{H_{n, m}\left(E_{02} ; x, y\right)}\right. \\
& \left.+\frac{1}{\delta_{1} \delta_{2}} \sqrt{H_{n, m}\left(E_{20} ; x, y\right) H_{n, m}\left(E_{02} ; x, y\right)}\right) \omega_{\text {mixed }}\left(f ; \delta_{1}, \delta_{2}\right) \\
\leq & \left(\frac{1}{\delta_{1}} \sqrt{\frac{b_{n}}{(n+1)}}+\frac{1}{\delta_{2}} \sqrt{\frac{b_{m}}{(m+1)}}\right. \\
& \left.+\frac{1}{\delta_{1} \delta_{2}} \sqrt{\frac{b_{n}}{(n+1)}} \sqrt{\frac{b_{m}}{(m+1)}}\right) \omega_{\text {mixed }}\left(f ; \delta_{1}, \delta_{2}\right)
\end{aligned}
$$

If we choose $\delta_{1}=\sqrt{\frac{b_{n}}{(n+1)}}$ and $\delta_{2}=\sqrt{\frac{b_{m}}{(m+1)}}$, we get

$$
\left|H_{n, m}^{*}(f(u, v) ; x, y)-f(x, y)\right| \leq 3 . \omega_{\text {mixed }}\left(f ; \sqrt{\frac{b_{n}}{(n+1)}}, \sqrt{\frac{b_{m}}{(m+1)}}\right),
$$

then the proof is being completed.

Theorem 5. If $f \in D_{B}\left(R_{b_{n} b_{m}}\right)$ with $D_{B} f \in B\left(R_{b_{n} b_{m}}\right)$, then for any $(x, y) \in R_{A B}$ and sufficiently large $n$ and $m$

$$
\left|H_{n, m}^{*}(f(u, v) ; x, y)-f(x, y)\right| \leq 3\left\|D_{B} f\right\|_{\infty}+\frac{64}{5} . \omega_{\text {mixed }}\left(D_{B} f ; \frac{b_{n}^{2}}{(n+1)}, \frac{b_{m}^{2}}{(m+1)}\right) .
$$

Proof. Applying Theorem which given [21] for B-Differentiable function, Lemma 2 ,( by taking in account (4) and (9)), (18), theorems defined by Bögel, we have

$$
\begin{aligned}
\left|H_{n, m}^{*}(f(u, v) ; x, y)-f(x, y)\right| \leq & 3\left\|D_{B} f\right\|_{\infty} \sqrt{\frac{b_{n}}{(n+1)} \cdot \frac{b_{m}}{(m+1)}}+\left[\sqrt{\frac{b_{n}}{(n+1)} \cdot \frac{b_{m}}{(m+1)}}+\frac{1}{\delta_{1}} \sqrt{\frac{29 b_{n}^{4}}{(n+1)^{2}} \cdot \frac{b_{m}}{(m+1)}}\right. \\
& \left.+\frac{1}{\delta_{2}} \sqrt{\frac{b_{n}}{(n+1)} \frac{29 b_{m}^{4}}{(m+1)^{2}}}+\frac{1}{\delta_{1} \delta_{2}} \cdot \frac{b_{n}}{(n+1)} \cdot \frac{b_{m}}{(m+1)}\right] \omega_{\text {mixed }}\left(D_{B} f, \delta_{1}, \delta_{2}\right) .
\end{aligned}
$$


If we choose $\delta_{1}=\frac{b_{n}^{2}}{(n+1)}$ and $\delta_{2}=\frac{b_{m}^{2}}{(m+1)}$ and take into account that $\frac{b_{n}}{(n+1)}<1$ for sufficiently large $n$ since $\lim _{n \rightarrow \infty} \frac{b_{n}^{2}}{n}=0$ then we get desired result.

\section{Some plots}

The following calculations are made by Maple17.

(1) We apply (11) for function $f(x, y)=x^{3}+y^{3}$ in the region $[0,5] \times[0,5]$, then we get the following graphics.Here $(n, m)=(10,10)($ red $),(n, m)=(20,20)$ (blue)

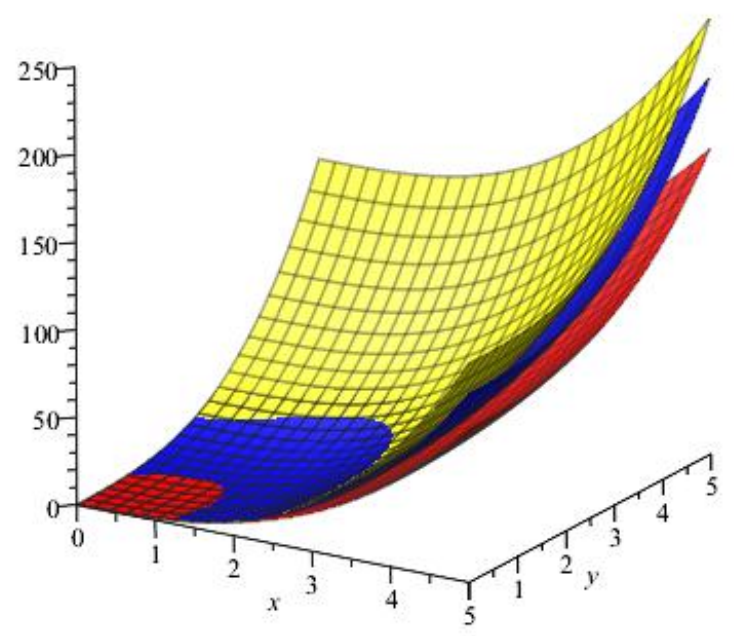

Fig. 1: The convergence of the $H_{n, m}(f(u, v) ; x, y)$ operator to the function $f(x, y)$.

(2) The convergence of $H_{n, m}^{*}(f(u, v) ; x, y)$ GBS operator for $(n, m)=(10,10)$ (red), $(n, m)=(20,20)$ (blue) to the function $f(x, y)=\left(x^{2}+y^{2}\right) \sin (\pi(x+y))$ (yellow) in the region $[0,1] \times[0,1]$ is pictorial as shown in Figure 2.

(3) For $(n, m)=(20,20)$, in the region $[0,5] \times[0,5]$, The convergence of $H_{n, m}(f(u, v) ; x, y)$ operator (red) and $H_{n, m}^{*}(f(u, v) ; x, y)$ GBS operator (blue) to the function $f(x, y)=(1+x+y) \sin (x+y)$ (yellow) is pictorial as shown in Figure 3.

Let $x=\left(x_{n}\right)$ and $y=\left(y_{n}\right)$ be sequences with limits $x_{0}$ and $y_{0}$ respectively. If

$$
\lim _{n \rightarrow \infty} \frac{\left|y_{n}-y\right|}{\left|x_{n}-x\right|}=0
$$

then it is said that $y$ converges faster than $x$ (see [27]). (Or equivalent $\lim _{n \rightarrow \infty} \frac{\left|x_{n}-x\right|}{\left|y_{n}-y\right|}=\infty$ ). And if $\lim _{n \rightarrow \infty} \frac{\left|y_{n}-y\right|}{\left|x_{n}-x\right|}=M<\infty$. than it is said that $x$ and $y$ have equivalent convergence. That means that there is any $n_{0}$ such that for all $n \geq n_{0}$ the inequality $\left|y_{n}-y\right| \leq M\left|x_{n}-x\right|$ holds. If $0<M<1$ then it is said that convergence of $y$ is better than $x$, and if $1<M<\infty$ then it is said that convergence of $x$ is better than $y$.(see [28]). Let $(X,\|\|)$ and $(Y,\|\|)$ be two function 


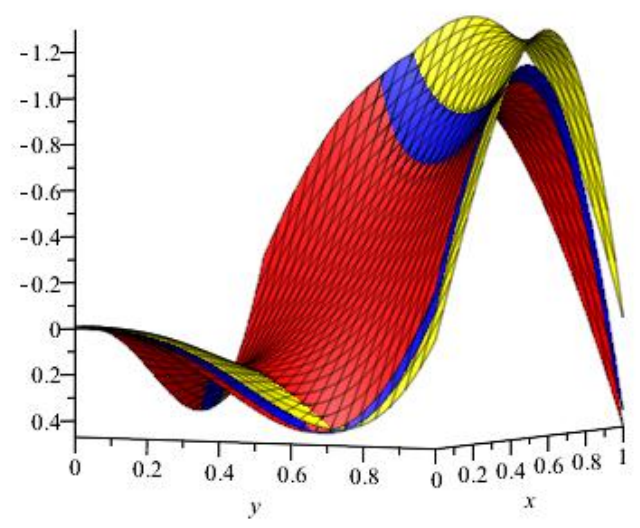

Fig. 2: The convergence of the $H_{n, m}^{*}(f(u, v) ; x, y)$ operator to the function $f(x, y)$.

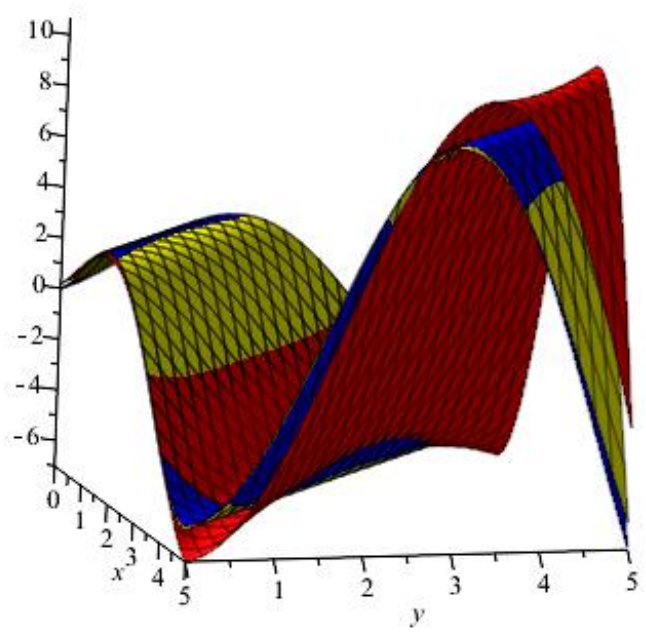

Fig. 3: The convergence of the $H_{n, m}(f(u, v) ; x, y)$ operator and $H_{n, m}^{*}(f(u, v) ; x, y)$ Gbs operator to the function $f(x, y)$.

spaces $\left(L_{n}\right)$ and $\left(M_{n}\right)$ be two linear positive operators sequences acting from $X$ into $Y$. And let these operators sequences approximate uniformly. If

$$
\lim _{n \rightarrow \infty} \frac{\left\|L_{n}(f ; x)-f(x)\right\|}{\left\|M_{n}(f ; x)-f(x)\right\|}=0
$$

(Or equivalent $\lim _{n \rightarrow \infty} \frac{\left\|M_{n}(f ; x)-f(x)\right\|}{\left\|L_{n}(f ; x)-f(x)\right\|}=\infty$ ) for any $f \in X$, then it is said that $\left(L_{n}\right)$ approximates faster than $\left(M_{n}\right)$. And if $\lim _{n \rightarrow \infty} \frac{\left\|L_{n}(f ; x)-f(x)\right\|}{\left\|M_{n}(f ; x)-f(x)\right\|}=K<\infty$, than it is said that $\left(L_{n}\right)$ and $\left(M_{n}\right)$ have equivalent approximate. If 
$0<K<1$ then it is said that approximate of $\left(L_{n}\right)$ is better than $\left(M_{n}\right)$, and if $1<M<\infty$ then it is said that approximate of $\left(M_{n}\right)$ is better than $\left(L_{n}\right)$.

We are ready to give the following example to compare approximation of $H_{n, m}(f ; x, y)$ and $H_{n, m}^{*}(f ; x, y)$ for some $(n, m)$ and $(x, y)$.

(4) We compare approximation of (11) and (23) to the function $f(x, y)=(1+x+y) \sin (x+y)$ in the region $[0,5] \times[0,5]$ for some $(n, m)$ and $(x, y)$ as following rate,

$$
A_{n, m}(x, y)=\left|\frac{H_{n, m}^{*}(f(u, v) ; x, y)-f(x, y)}{H_{n, m}(f(u, v) ; x, y)-f(x, y)}\right|
$$

and then we get the following numerical table.

Table 1: Error bounds at different $n$ values for $H_{n, m}(f ; x, y)$ and $H_{n, m}^{*}(f ; x, y)$ GBS operator.

\begin{tabular}{lcccc}
\hline$(\mathrm{n}, \mathrm{m})-(\mathrm{x}, \mathrm{y})$ & $(1,1)$ & $(2,2)$ & $(3,3)$ & $(4,4)$ \\
\hline$(10,10)$ & 0.2748334033 & 0.0327836043 & 0.0096505536 & 0.4496459206 \\
$(20,20)$ & 0.01368673105 & 0.03275994979 & 0.04873530553 & 0.1256181241 \\
$(50,50)$ & 0.003614794524 & 0.0031655557953 & 0.03180114071 & 0.006245528269 \\
\hline
\end{tabular}

From the table we see that, approximation of (23) is better than (11) for the function $f(x, y)$.

\section{Competing interests}

The authors declare that they have no competing interests.

\section{Authors' contributions}

All authors have contributed to all parts of the article. All authors read and approved the final manuscript.

\section{References}

[1] Bernstein, S.N.: Demonstration du theor'eme de Weierstrass basee sur le calcul des probabilit'es, Commun. Soc. Math. Kharkow (2) 13 (19121913) 12.

[2] A.Attalienti and M. Campiti, Bernstein type operators on the half line, Czec. Math. J., 52(127)(2002), no.4, 851-860.

[3] S. Lewanowicz and P. Wonźy, Generalized Bernstein polynomials, BIT Numerical Mathematics44 (2004), 6378.

[4] G.M. Phillips, Bernstein polynomials based on the q-integers, Annals of Numerical Mathematics4 (1997), 511518.

[5] I. V. Kantorovich, Sur certains dèvelopments suviant les polynōmes de la forme de S.Bernstein, I.H.C.R. Acad. URSS (1930), 563-568, 595-600.

[6] I. Chlodowsky. Sur le dĕveloppment des fonctions dĕfines dans un interval infinien sĕries de polynŏmes de S. N.Bernstein, Compositio Math., 4(1937), 380-392.

[7] A. D. Gadjiev, R.O.Efendiev, E.Ibikli, Generalized Bernstein-Chlodowsky Polynomials, Rocky Mountain Journal of Mathematics,V28, No:4,(1998),1267-1277. 
[8] E. A. Gadjieva and E. Ibikli. The Weighted Approximation by Bernstein-Chlodowsky Polynomials. Indian J. Pure Appl. Math., 30(1): January (1999), 83-87.

[9] E. Ibikli, On Approximation by Bernstein-Chlodowsky Polynomials. Mathematica Balkanica. New Ser. Vol. 17(3-4), (2003), 259265

[10] A. Attalanti and M. Campiti, Bernstein type operators on the half line, Czec. Math. J., 52(127)(2002), no.4, 851-860.

[11] A. Habib and A. Wafi. Degree of Approximation of Functions by Modified Bernstein Polynomials On Unbounded Interval. Indian J.of Pure and Appl. Maths. 8(6),(1977),691-695.

[12] N. Ispir, A. Aral and O. Dogru. On Kantorovich Process of A Sequence Of The Generalized Linear Positive Operators. Numerical Functional Analysis and Optimization, 29(56): (2008), 574589.

[13] Bögel, K. Mehrdimensionale Differentitation von Funktionen mehrerer Veränderhicher, J. Reine agnew. Math., 170, (1934) 197217.

[14] Bögel, K.Über die mehrdimensionale Differentitation, Integration and beschränkte variation, J. Reine agnew. Math., (1935) 173, 5-29.

[15] Bögel, K. Über die mehrdimensionale Differentitation, Jber. DMV, 65, (1962) 45-71.

[16] Badea, C. , Cottin, C. Korovkin-type theorems for Generalized Boolean Sum operators, Colloquia Mathematica Sociekatis Janos Bolyai, 58, Approximation Theory, Kecskemet(Hungary), (1990) 51-68.

[17] Badea, C. ,Badea, I. , Gonska, H. H. A test function theorem and approximation by pseudopolynomials, Bull. Austral. Math. Soc.,34, (1986) 53-64.

[18] Badea, I. Modul de continuitate in sens Bögel şi unele applicatii in approximarea printr-un operator Bernstein, Studia Univ. "Babeş-Bolyai" Ser. Math-Mech., 18(2), (1973)69-78, (Romanian).

[19] Bărbosu, D. GBS operators of Schurer-Stancu type., Annals of Univ. of Craiova, Math. Comp. Sci. Ser. 2(30), (2003) 34-39.

[20] Pop, O. T. and Farcaş, M. Approximation of B-continuous and B-differentiable functions by GBS operators of Bernstein bivariate polynomials. 3(7), (2006) 1-9.

[21] Pop, O. T. Approximation of B-differentiable functions by GBS operators. Analele Univ. Oradea. Fac. Mathematica, Tom. XIV, (2007) 15-31.

[22] Pop, O. T. On the approximation by GBS operators of exponantial type. Studia Univ. "Babeş-Bolyai", Mathematica, 1(LII), (2007) 99-110.

[23] Muraru , C-V. and Niminet, V. On a bivariate Generalized Boolean Sum type operator. Univ. Din. Bacău Studii Cercetări Ştiinfice. Seriai Mathematică, 18, (2008)153-160.

[24] Volkov, V. I.On the convergence of sequences of linear positive operators in the space of continuous functions of two variable, Math. Sb. N. S. 43(85) (1957) 504 (Russian).

[25] Nicolescu, M. Analizä matematicä, II, Editura Didacticä şi Pedagogica. Bucureşti, 1980(Romanian).

[26] O. T. POP and M. D. FĀRCAŞ. About The Bivariate Operators Of Kantorovich Type Acta Math. Univ. Comenianae, Vol. LXXVIII, 1(2009), 43-52.

[27] C. Brezinski. Convergence acceleration during 20 th century. J. Comput. Appl. Math. 122(2000)1-21.

[28] B. S. Theodore Ho Hsu. A non-linear transformation for sequences and integrals. Thesis master science. Graduate Faculty of Texas Thechnological College, 1968. 